\title{
Structures of Planar Defects in ZnO Nanobelts and Nanowires
}

\author{
Yong Ding and Zhong Lin Wang \\ School of Materials Science and Engineering, Georgia Institute of Technology, Atlanta, \\ GA 30332-0245 USA
}

Quasi-one-dimensional (1D) nanostructures, such as nanowires, nanobelts and nanorods, are the forefront materials for nanotechnology. [1] To date, such nanostructures have been synthesized for a wide range of semiconductors and oxides, and they are potential building blocks for fabricating numerous nano-scale devices. 1D $\mathrm{ZnO}$ nanostructures, due to its unique semiconducting, piezoelectric, and bio-safe properties, have received wide attention. From structure point of view, a common characteristic of $\mathrm{ZnO}$ nanostructures is that they are mostly dislocation-free. However, planar and point defects do frequently exist in such nanostructures. The objective of this work is to present detailed electron microscopy study about the structures of planar defects, such as stacking faults, twins, inversion domain walls that existed in $1 \mathrm{D} \mathrm{ZnO}$ nanostructures. These planar defects are important for understanding the growth mechanism and relevant physical and possibly chemical properties of $1 \mathrm{D} \mathrm{ZnO}$ nanostructures.[1]

Figure 1(a) shows a typical $\mathrm{ZnO}(01 \overline{1} 3)$ twin structure [2]. Its SAED pattern is displayed in Fig. 1(b), which is composed of two sets of diffraction spots that have symmetrical geometrical distribution but possibly variable intensity.

Figure 2(a) is a low magnification TEM image of another $\mathrm{ZnO}[01 \overline{1} 0]$ growth nanobelt. There are two different basal-plane stacking faults exist in Fig. 2(a), the top one is the $I_{1}$ stacking fault. A HRTEM image of the bottom defect is shown in Fig. 2(b). In the defect area, the stacking sequence can be described as ...ABABCACAC ... It can be classified as an intrinsic type $\mathrm{I}_{2}$ basalplane stacking fault science it is equivalent to extracting two periodic layers and a $2 / 3[01 \overline{1} 0]$ translation displacement has been measured across the defect.

Besides basal-plane stacking faults, we also observed prismatic-plane stacking faults in wider $\mathrm{ZnO}$ nanobelts $(\sim 1 \mu \mathrm{m})$. In order to determine the structural nature of the defect in the nanobelts, a series of dark-field images have been recorded as shown in Fig. 3. The defect contrast can be seen clearly in Fig. 3(a) and (b), which are the corresponding bright-field and dark-field images under two-beam condition with the $g=(02 \overline{2} 0)$. The diffraction condition is inserted in Fig. 3(b). By further tilting the nanobelt, we get two extinguishing conditions of the defects as shown in Fig. 3(c) and (d). The displacement crossing the stacking faults can be determined as $1 / 6<02 \overline{2} \overline{3}>$.

\section{References:}

[1] Z.L. Wang, Nanowires and Nanobelts, Vol. I: Metal and Semiconductor, Nanowires, Vol. II: Nanowires and Nanobelts of Functional Materials, Kluwer Academic Publisher, New York, (2003).

[2] Y. Ding and Z.L. Wang, Micron, 10.1016/j.micron.2008.10.008.

[3] Y. Ding and Z.L. Wang, J. Phys. Chem. B 108, 12280 (2004). 


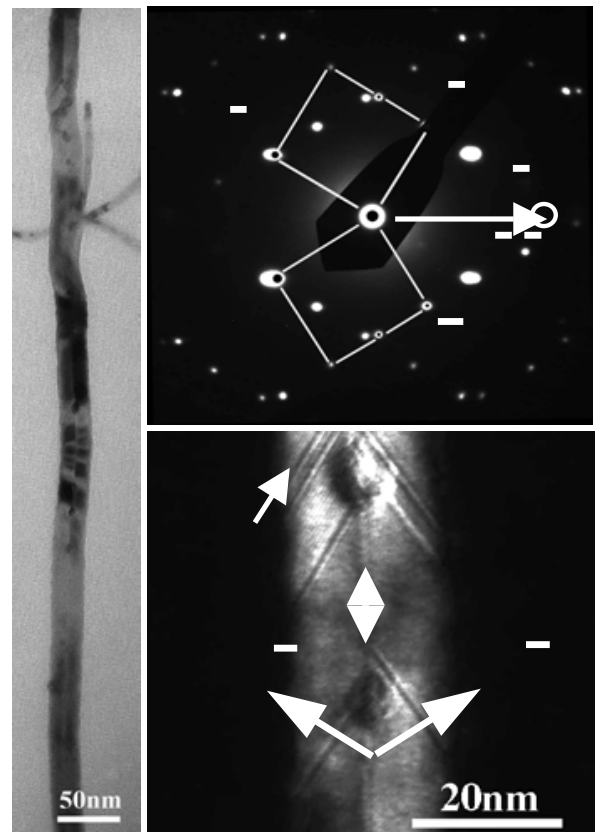

Fig. 1 (a) and (b) TEM image and corresponding SAED pattern of a $\mathrm{ZnO}$ nanobelt that has a $(01 \overline{1} 3)$ twin parallel to the growth direction. (c) A dark-field TEM image showing the existence of stacking faults in the nanobelt.

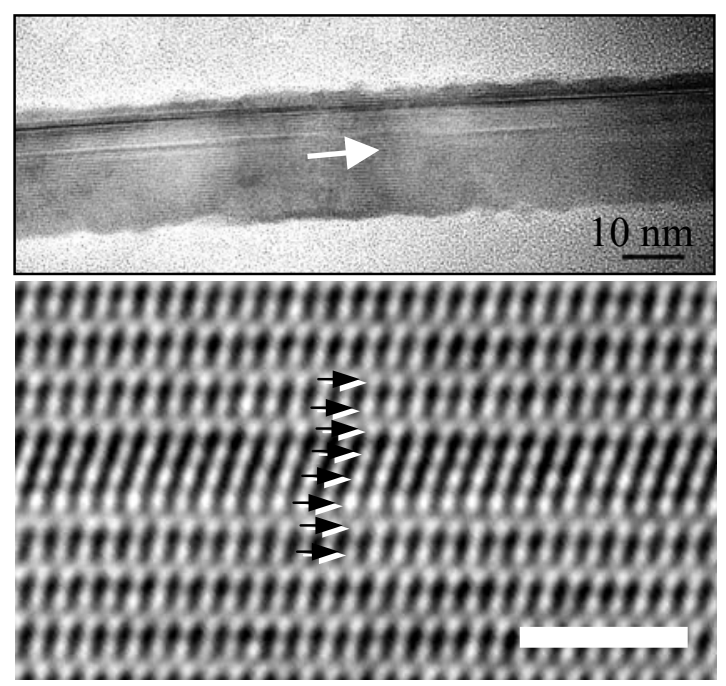

Fig 2. Low-magnification (a) and HRTEM (b) images of a $[01 \overline{1} 0]$ growth $\mathrm{ZnO}$ nanobelt with incident electron beam along [2 $\overline{1} \overline{1} 0]$ direction. The defect in (b) belongs to the type $\mathrm{I}_{2}$ stacking

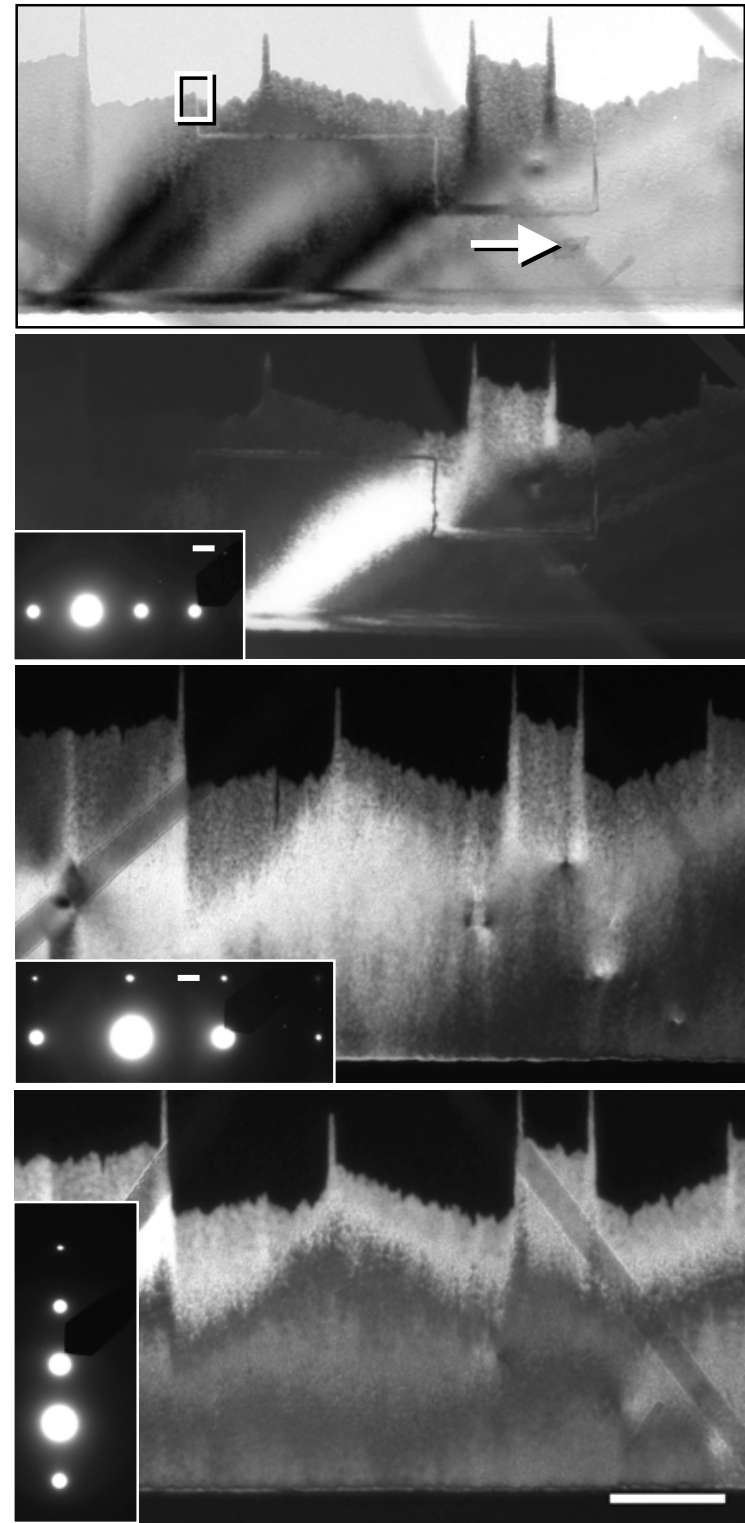

Figure 3 Bright-field (a) and dark-field (b) images of a [01 $\overline{1} 0]$ growth $\mathrm{ZnO}$ nanobelt recorded under two-beam condition with $\mathrm{g}=$ $(02 \overline{2} 0)$. Prismatic and basal plane stacking faults coexist in the belt. In the dark-field images recorded using $g=(\overline{2} 110)(c)$ and $0002(d)$, the contrast of the defect vanishes. 